\title{
Study on Dynamic and Non-destructive Ultrasonic Echo Detection Method of Plant Stem Water Content
}

\author{
Lyu Danju ${ }^{1,2}$, Shi Xinling ${ }^{11}$, Wang Xia ${ }^{1}$, Dong Yi' ${ }^{1}$, Wang Yueming ${ }^{1}$, Qing Zhiyi ${ }^{2}$,Liu Huangfei ${ }^{2}$ \\ ${ }^{1}$ Information School, Yunnan University, Kunming, Yunnan 650091, China \\ ${ }^{2}$ School of Computer and Information, Southwest Forestry University Kunming, Yunnan 650224, China
}

Keywords: stem water content, ultrasonic pulse echo detection, dynamic detection.

\begin{abstract}
Stem water content is an important index of plant water balance. A new method based on changes in ultrasonic properties of stem was used to monitor stem water content in this paper, because the propagation of ultrasonic pulse echo signal and its attenuation characteristics in the material are closely related to the structure of the material. Ultrasound propagation echo signal was measured with an ultrasonic emission analysis system in the plant stem. The study showed that the increase of stem water content in the Populus simonii stem could cause the average amplitude, the root mean square (RMS) and the peak of value of the ultrasonic echo signal decreased obviously. The analysis of ultrasonic properties provides a new method for the detection of stem water content of trees and may be used to monitor plant water balance.
\end{abstract}

\section{Introduction}

The absorption, transporting and losing of water in plant are it's normal life activities to maintain the water balance[3]. Changes in the water content of the plant is often used as an indicator of water balance, which provides an important indicator for the diagnosis of plant physiological drought by directly measuring the moisture content of the stem plant [1],[4].

Currently direct detection method uses plant stem water content are: drying method, $\gamma$-ray method [7], nuclear magnetic resonance spectroscopy [5], Computer tomography [9], and dielectric constant resistance method[4]. However, taking the above detection means will cause varying degrees of damage to the plant. Nuclear magnetic resonance, computer tomography technology, drying method and $\gamma$-ray detection method, requiring stem samples truncated from trees, undermine the physiological activities of plants, which are unable to achieve on-line measurement. The resistance measurement has some limitations, because the absolute moisture content of living tissue with the resistance value is not a single-valued function [2]. Currently, time domain reflectometry (TDR) achieved quite good results for detection of water content in stems of standing trees [6],[8]. But this method still needs to insert probes into the plant stem to detect that causes some damage to the plant itself, affects plant physiological activity and finally generates detection errors of dynamical detection of water content in the stem.

a Corresponding author : xlshi@ynu.edu.cn 
Since the propagation and attenuation of ultrasonic echo signal in the material are closely related to the composition and the structure of the material[1], this paper presents a dynamic non-destructive method of plant stem water content with ultrasonic echoes by analysing the average amplitude and other time features of the ultrasonic signal to detect water content of the plant stem dynamically.

\section{Detection Principles}

According to the ultrasonic propagation in elastic media, energy attenuation occurs that is the sound pressure amplitude exponentially decreases with the increasing ultrasonic propagation distance $\mathrm{x}$, which can be stated by the expression of $\mathrm{p}(\mathrm{x})=\mathrm{p}_{0}{ }^{-\mathrm{Ax}}$, where $\mathrm{p}_{0}$ is the initial pressure amplitude value, $\mathrm{A}$ is the attenuation coefficient [1]. Different elastic media holds different attenuation characteristics, such as the acoustic impedance of water at $20^{\circ} \mathrm{c}$ is $1.48 \times \mathrm{MPa} \mathrm{s}-1$, the air acoustic impedance is 0.0004 $\times \mathrm{MPa}$ s-1. Therefore, the impedance of elastic media will vary when the constituent of it changes.

The average water content of the plant stem will changes at different conditions. Generally, it is about $50 \%$ for plants. When the loss of water by transpiration is greater than the amount of absorption by the roots of plants, plant water content decreased, resulting in plant stems water content also decreased. Conversely, when the soil fills with moisture, roots absorbs sufficient moisture and the stems water content increase and transport to the other parts of the plant through the root pressure and transpiration. In words, plant stem water content changes can be reflected by the mean, RMS and peak-peak values of ultrasound.

\section{Detection Plant stem water content by Ultrasonic Pulse Echoes}

\subsection{Equipment and materials}

An ultrasound apparatus, ultrasonic pulse echo detection system for stem water content, is designed as shown in Figure 1. It consists of a transmitting and receiving ultrasonic probe (a non-metallic ultrasound $250 \mathrm{KHz}$ probe), a non-metallic ultrasonic pulse transmitting and receiving device (Shantou Ultrasonic CTS-8077PR), a Nextkit data acquisition module based on LabVIEW (Global China CNC Nextkit) and a PC machine.

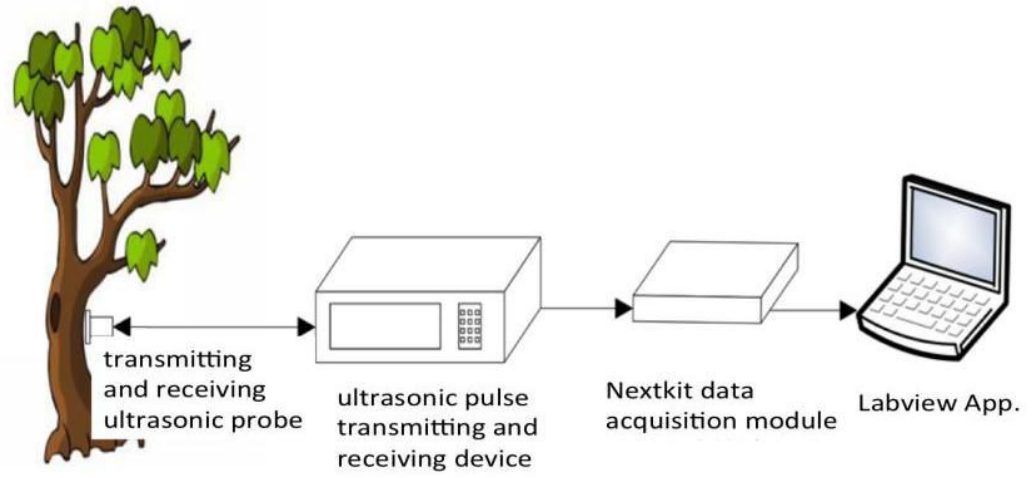

Figure 1. Ultrasonic Pulse Echo Detection System for Plant Stem Water Content

One 15-year-old Poplar leaflet (Populus simonii Carr) at the Southwest of Forestry University in Kunming, China was used as the test materials. The diameter at the breast height $(1 \mathrm{~m}$ from the ground level) is $12.7 \mathrm{~cm}$. The test data are $4^{\text {th }}, 5^{\text {th }}$ and $7^{\text {th }}$ of June in 2015 . Before $4^{\text {th }}$ of June, Kunming lasted for 20 days without rainfall. From $5^{\text {th }}$ to $7^{\text {th }}$ of June, Kunming last continuous rainfall. The test temperature is $20^{\circ} \mathrm{C}$. 


\subsection{Data Acquisition}

The detection system uses ultrasonic echo technique to detect water content in plant stem. Firstly, the ultrasonic pulse transmitting device generate $1 \mathrm{KHz}$ narrow pulses with $1000 \mathrm{~ns}$ pulse width, which makes the $250 \mathrm{kHz}$ ultrasonic probe to generate ultrasonic waves; and then, the probe is placed at the plant stem with a sufficient amount of coupling agent which make ensure that the probe can fully be coupled with the plant stem; after that, the ultrasonic waves spread in the plant stem in the axial direction. The absorption of the ultrasonic energy by the plant stem leads to reduction of ultrasonic energy. Therefore, when the ingredient of the plant stem changes to intent to absorb more energy, the energy attenuation of ultrasounds increases gradually. Finally, the ultrasonic echo signals are received via a receiving probe, which will be stored and displayed on a PC via the data acquisition card on Nextkit and LabVIEW platform with $1 \mathrm{MHz}$ sampling rate and 1000 points sampling depth.

\subsection{Data Pre-processing}

The ultrasonic echo data are pre-processed before analysing on Matlab. For each ultrasonic record is about $5 \mathrm{~s} \sim 10 \mathrm{~s}$ filled with a lot of noise. In order to reduce the detection error caused by disturbance, the formula (1) is adopted to select effective ultrasonic echo pulses when the EH of an ultrasonic echo pulse is bigger than settled EHs.

$$
E H>E H_{S}
$$

The EH considers 2 important factor: energy(ENE) and high-order difference (HOD) that presents the high frequency feature in time domain. $\mathrm{EH}_{\mathrm{S}}$ is the threshold of EH. It presents as following steps: Step 1. Compute energy (ENE) and the absolute sum of order-n difference (HOD).

Step2. Select a weighting factor $\mathrm{w}$ within $[0,1]$ to compute a new curve $\mathrm{EH}=\mathrm{w}^{*} \mathrm{ENE}+(1-\mathrm{w})^{*} \mathrm{HOD}$. Step3. Find a ratio to compute the threshold of EH to determine the effective. The threshold is equal to:

$$
\mathrm{EH}_{\mathrm{s}}=\mathrm{EH}_{\min }+\left(\mathrm{EH}_{\max }-\mathrm{EH}_{\min }\right) * \mathrm{r}
$$

Step4. Select the effective ultrasonic data using the formula(1).

The above method involves three parameters to be determined: $n, w, r$. In this paper, the values of these parameters are $n=4, w=0.76$, and $r=0.012$. However, these values vary with data sets. It is always advisable to have these values tuned by using the target data set for a more robust result.

\section{Results}

The ultrasonic data of 3 days about 10s are pre-processed using the above method to get the effective data as shown in Figure2. Each test effective data are average to one ultrasonic echo pulse as shown in Figure 3 for further analysis. This paper analyses the mean, variance, RMS and the peak to peak values of the one ultrasonic echo pulse as stated in Table 1.

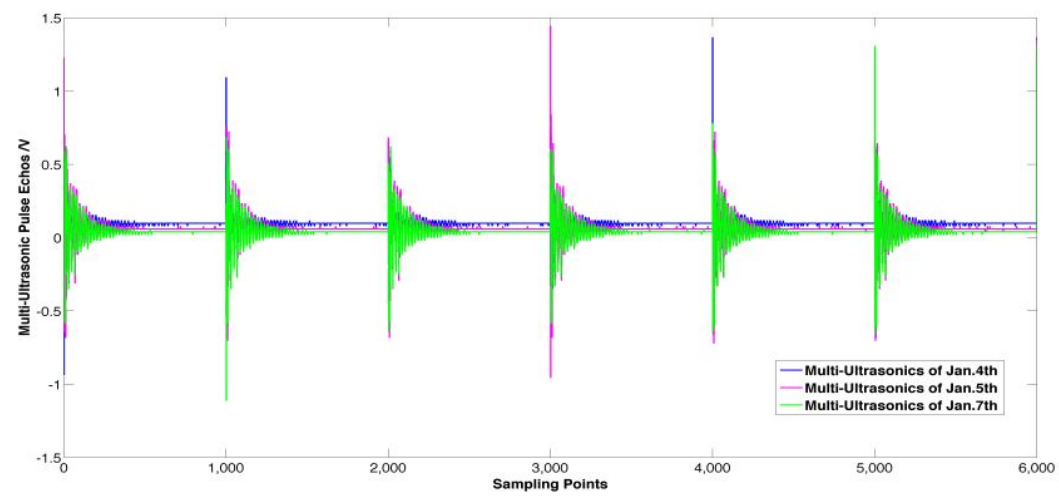

Figure 2. Multi-Ultrasonic Pulse Echoes in the Stem of Populus simonii 


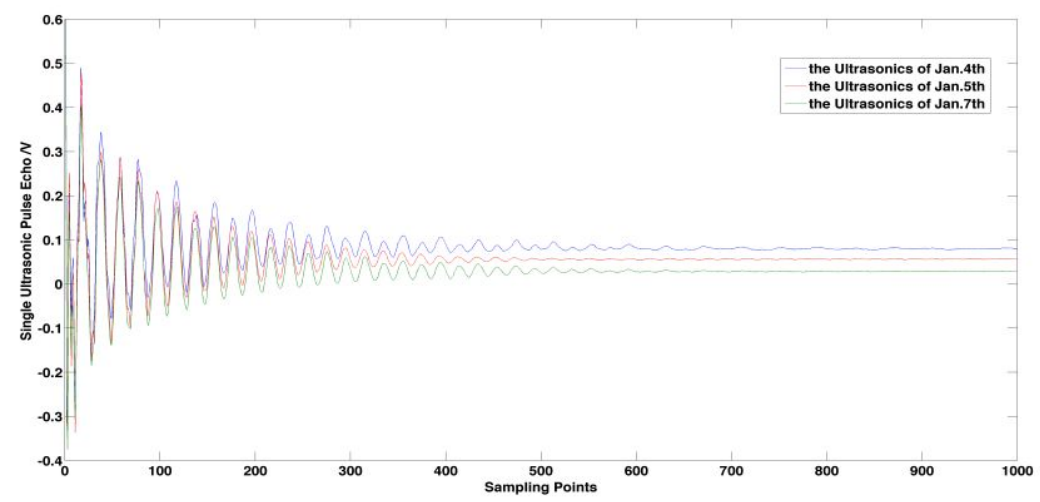

Figure 3. Single Ultrasonic Pulse Echo in the Stem of Populus simonii

Table 1. the Analysis of Ultrasonic Pulse Echo in the stem of Populus simonii

\begin{tabular}{|c|c|c|c|}
\hline Test Date & June 4 & June 5 & June 7 \\
\hline Mean & 0.0731 & 0.0595 & 0.0326 \\
\hline Variance & 0.0041 & 0.0034 & 0.0032 \\
\hline RMS & 10.6957 & 6.9415 & 4.2962 \\
\hline Peak to Peak & 0.8959 & 0.8127 & 0.7058 \\
\hline
\end{tabular}

\section{1 the Analysis of Ultrasonic Pulse Echo Envelope and Plant Stem Water Content}

Figure 3 shows that the envelopes of ultrasonic echo signal in plant stem attenuate exponentially, the expression of $\mathrm{p}(\mathrm{x})=\mathrm{p}_{0}{ }^{-\mathrm{Ax}}$. The attenuating exponent is closely related to the water content in the plant stem. The exponent A1 of June 4 is the maximum in those of all the 3 days for without rainfall, which followed by A2 of June 5 for starting to rain, the minimum is A3 for lasting continuous 3-day rainfall, namely A1 $>$ A2 $>$ A3. The results indicate that the smaller the exponent is, the more the water content of same stem plant is.

\section{2 the Analysis of Ultrasonic Pulse Echo Means and plant stem water content}

The means in Table 1 shows that on June 4 ,rain-free, the mean of the ultrasonic is maximum, which indicates that the ultrasonic signal propagation in plant stem met the smallest impedance, leading the minimum energy attenuation; on June 5 ,rain for 1 day, the mean significantly reduced down to $0.0269 \mathrm{v}$; on June 7 , rain for 3 days, the mean continues to decline, but the decline was significantly better than June $5,0.0136 \mathrm{v}$ to that of on June 5 . The above data show that the changes of the water content in plant stem can be reflected by the mean of ultrasonic pulse echo signals. When the plant stems water increases, the energy absorption of ultrasonic pulse echo by the stem gets more, so that the ultrasonic echo attenuation increases, eventually leading to the reduction of the mean ultrasonic pulse echo signal.

\section{3 the Analysis of Other Properties of Ultrasonic Pulse Echo and Plant Stem Water Content}

The RMS, variance and amplitude from peak to peak of the first wave may reflect the propagation of ultrasonic energy. The greater of these parameters are, the smaller the impendence of the ultrasonic is. According to Table 1, on June 4 rain-free the RMS, the variance and the value of peak to peak, which presents the ability to radiate the ultrasonic energy, are the maximum in the 3 test days, indicating that 
on June4 the stem has the maximum ultrasonic energy, hindered minimum attenuation with the greatest outward radiation energy capacity. After one day raining the significant decreasing of RMS and variance manifests the reducing of the ultrasonic energy and the ability to radiate energy also declined. After 3 days raining, the 3 parameters continuously decline but not so sharply as they dose in the first two test days.

\section{Discussion}

A plant stems ultrasonic echo detection system, a no-destructive dynamic detection, was designed for detecting the changes of water content in living plant stems as a new and effective means. In this paper, the stems of plants of ultrasonic pulse echo signal only from the time domain analyses the energy mean, variance, peak to peak and RMS future timely frequency from the frequency domain to the angle of the echo signal as a basis for further analysis of the signal characteristics relationship between moisture changes. However, due to the ultrasonic echo signal attenuation is very strong in plant stem body, the first echo signal in the test data is not obvious. Therefore, the ultrasonic probe depth analysis is not discussed here.

\section{Acknowledgement}

This work is partially supported by the National Natural Science Foundation of China Grant \#61261007 to Y.F. Zhang, \#61462078 to Y.Zhang, the Key Program of Yunnan Natural Science Foundation the Grant \#2013FA008 to Y.F. Zhang and the Yunnan Natural Science Foundation the Grant \#2013FB048 to Y.F. Zhang.

\section{References}

1. H.JianKai, in:Ultrasonic Testing Principles and Methods, chapter,4, University of Science and Technology of China Publisher (1983).

2. S. Zhecun, L. Yingzhong: Journal of Northeast Forestry University,Vol.22(1) (1994), p. 113-116.

3. Y. Shuwen: Plant Physiology Communications Vol. 2 (1958), p.1.

4. Z. Yandong, W. Hilan, H. Peijin, et al.: Scientia Silvae Sinicae Vol. 46(11) (2010), p. 179-183.

5. G.F. Byrne, M.D. Fenn, M.I. Burgar M I:. Agricultural and forest meteorology Vol. 38(4) (1986), p. 307-317.

6. J.Constantz, F. Murphy: Journal of Hydrology Vol. 119(1) (1990), p. 31-42.

7. W.R.Edwards, P.G.Jarvis: Plant, Cell \& Environment Vol. 6(3) (1983), p. 255-260.

8. A. Nadler, E. Raveh, U. Yermiyahu,et al.: Soil Science Society of America Journal Vol. 67(2) (2003), p. 437-448.

9. A.Raschi, R.Tognetti, H.W.Ridder,et al.: Plant, Cell \& Environment Vol.18(5) (1995), p.545554. 